\title{
SARS-CoV-2 Sequel: Pulmonary Mucormycosis with a Mycotic Aneurysm in a Transplant Recipient
}

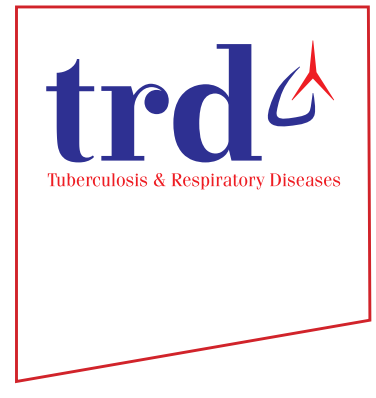

\author{
Klein Dantis, M.S., D.N.B. ${ }^{1}{ }^{(\mathbb{D})}$, Vinay Rathore, M.D., D.M. ${ }^{2}$, Nitin Kumar Kashyap, M.S., M.Ch. ${ }^{1}$, \\ Nilesh Gupta, M.D. ${ }^{3}$, Sajal De, M.D. ${ }^{4}$ and Subrata Kumar Singha, M.D. ${ }^{5}$ \\ Departments of ${ }^{1}$ Cardiothoracic Surgery, ${ }^{2}$ Nephrology, ${ }^{3}$ Radiodiagnosis, ${ }^{4}$ Pulmonary Medicine, and ${ }^{5}$ Anesthesiology, All India \\ Institute of Medical Sciences, Raipur, India
}

Mucormycosis is an invasive disease, and management is challenging in patients with severe acute respiratory syndrome coronavirus 2 (SARS-CoV-2) disease with transplant recipients due to data paucity. Mycotic aneurysm in the pulmonary artery from SARS-CoV-2 sequelae is uncommon with no reports to date.

A 46-years old male with a history of SARS-CoV-2 disease, diagnosed 8 weeks ago, presented with right-sided chest pain, cough, and hemoptysis for 1 week. Eleven years ago, he had a 6-month history of being treated for pulmonary tuberculosis., and had a right renal transplant 8 years ago, for which he is receiving oral prednisolone ( $20 \mathrm{mg} /$ day), mycophenolate mofetil (1,000 mg/day), and tacrolimus ( $2 \mathrm{mg} /$ day). No cavitary lesions were reported on contrast enhanced computed tomography (CECT) at the time of diagnosis of SARS-CoV-2 disease and on the 14th day from the time of diagnosis (Figure $1 \mathrm{~A}, \mathrm{~B})$. Informed consent was obtained from the patient for the purpose of publication.

He had tachycardia with oxygen saturation of $94 \%$ in room air with decreased right breath sound on examination. Blood investigations were regular, and the renal function test was above normal. Chest X-ray showed increased right bronchovascular markings (Figure 2A). CECT showed a cavitary lesion with eccentric ballooning and a contrast uptake with air-fluid level (Figure 2B). A 3-D reconstructive image demonstrated abnormal saccular dilatation of the basal segmental branch of the pulmonary artery suggestive of an aneurysm impending to rupture in the right lower lobe (Figure 2C). A sputum smear was negative for acid-fast bacilli as well as for fungal culture. Bronchoscopy and bronchoalveolar lavage did not reveal any abnormality, and subsequently, a computed tomography-guided transthoracic biopsy from the wall of the cavitary lesion showed a mycotic structure consistent with mucormycosis. Thus, a diagnosis of pulmonary mucormyco-
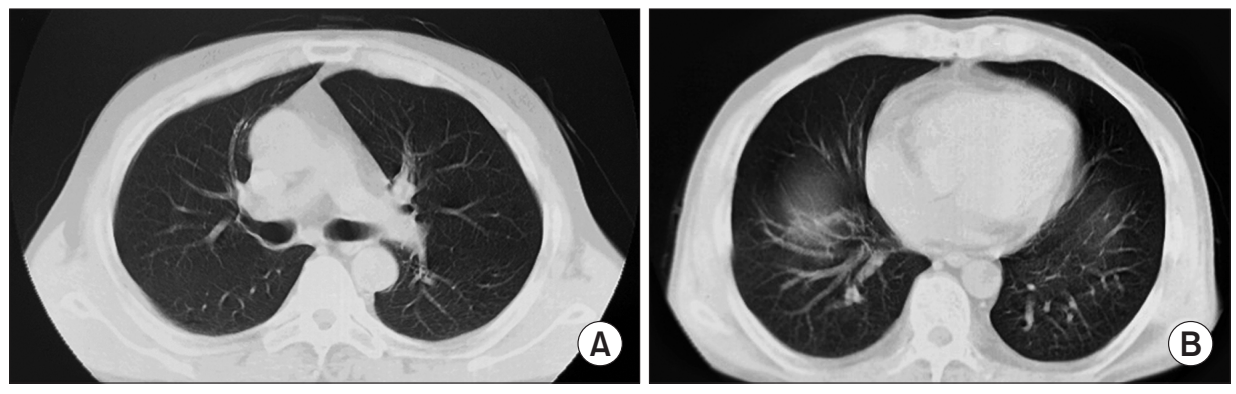

Figure 1. Contrast enhanced computed tomography thorax at and below the level of carina at the time of diagnosis of severe acute respiratory syndrome coronavirus 2 disease with no cavitary lesion.

Address for correspondence: Klein Dantis, M.S., D.N.B.

Department of Cardiothoracic Surgery, All India Institute of Medical Sciences, Raipur, Chhattisgarh 492099, India

Phone: +91-9886044143, E-mail: drkleindantis86@gmail.com

Received: Jun. 29, 2021, Revised: Jul. 24, 2021, Accepted: Aug. 1, 2021, Published online: Aug. 3, 2021

@(c) it is identical to the Creative Commons Attribution Non-Commercial License (http://creativecommons.org/licenses/by-nc/4.0/) 

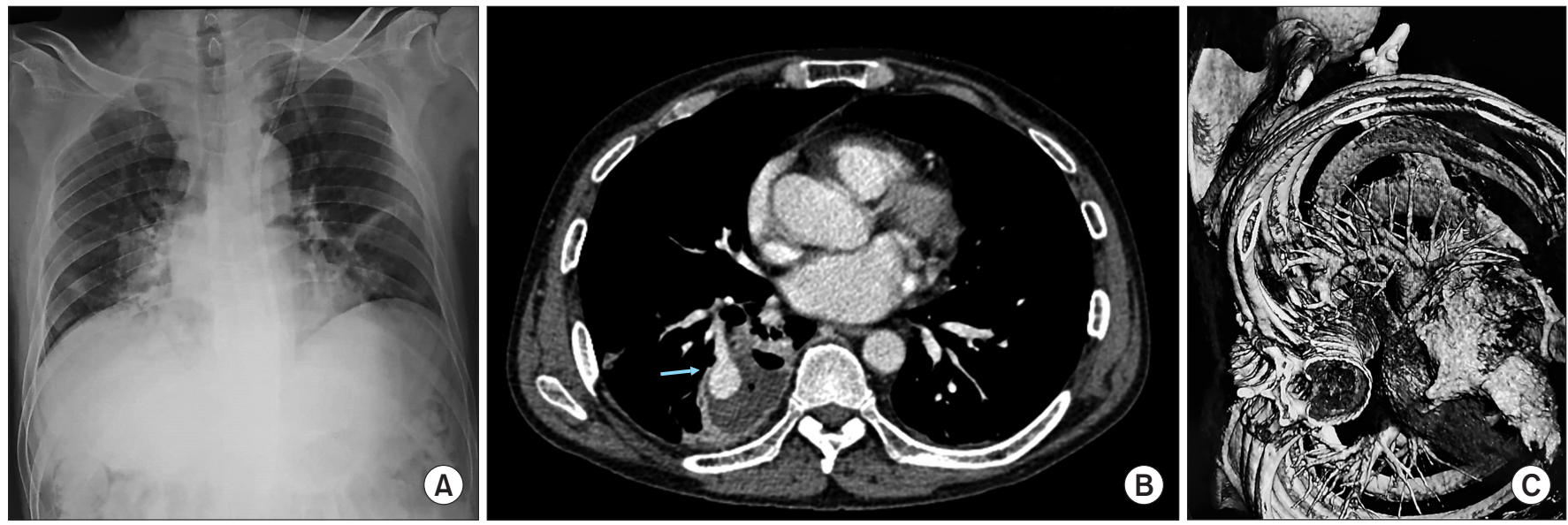

Figure 2. (A) Chest x-ray PA view showing increased right bronchovascular markings. (B) Cavitary lesion in the right lower lobe with eccentric ballooning and a contrast uptake with an air-fluid level. (C) A 3-D reconstructive image showing aneurysm of the basal segmental branch of the right pulmonary artery.

sis with a mycotic aneurysm secondary to SARS-CoV-2 sequel was made.

Preoperatively for four days, he received treatment with intravenous liposomal Amphotericin B, $300 \mathrm{mg} /$ day (minimum cumulative dose of $1 \mathrm{~g}$ ), followed by right lower lobectomy under double-lumen intubation. Postoperatively intravenous antifungal was continued till the cumulative dose of $5 \mathrm{~g}$ with a simultaneous continuation of postrenal transplant immunosuppressants Prednisolone (20 mg/day) and Tacrolimus (2 $\mathrm{mg} /$ day), which was continued further. The patient recovered well postoperatively. The gross specimen showed a cavitary lesion with blackish-brown material, confirming mucormycosis histopathologically (Figure 3A, B).

Rasmussen and mycotic aneurysm have similar risk factors and are clinically indistinguishable. However, the former has been associated with pre-existing cavitary lesion, while the latter in our case presented after eight weeks of SARS-CoV-2 infection with tissue biopsy of mucormycosis ${ }^{1}$. Suspected etiology was the weakening of the arterial wall due to intimal defect leading to aneurysm formation, and the predominant factor was the direct invasion from the adjacent source of sepsis surrounding the aneurysm. CECT obtained at the time of diagnosis of SARS-CoV-2 did not detect any cavitary lesions, was the differentiating feature of pulmonary mucormycosis after solid organ transplant with long term immunosuppressants.

Successful management of pulmonary mucormycosis with underlying solid organ transplant is a multimodal approach ${ }^{2}$. The implementation of the following principles-early diagnosis, reversal of the predisposing factor, antifungal therapy, and surgical intervention was necessary for better outcome and survival. Antifungal provides salvage therapy, while surgical resection prevents further tissue invasion and parenchymal damage.
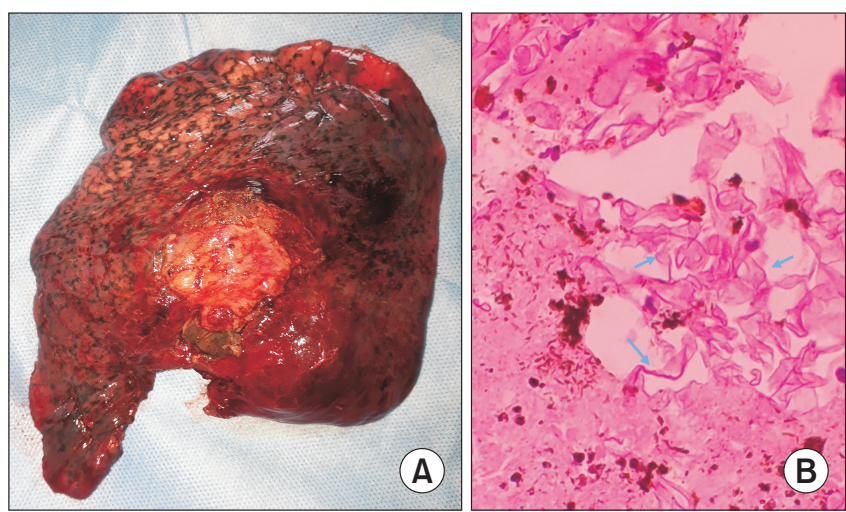

Figure 3. (A) Gross specimen of the mucor lung. (B) Microscopy showing aseptate hyphae suggestive of mucormycosis.

\section{Authors' Contributions}

Conceptualization: Dantis K. Methodology: Rathore V. Formal analysis: De S. Data curation: Kashyap NK. Software: Gupta N. Validation: Singha SK. Investigation: De S. Writing original draft preparation: Dantis K. Writing - review and editing: all authors. Approval of final manuscript: all authors.

\section{Conflicts of Interest}

No potential conflict of interest relevant to this article was reported. 


\section{Funding}

No funding to declare.

\section{References}

1. Cajigas-Loyola SC, Miller RL, Spieler B, Carbonella G. My- cotic pulmonary artery aneurysm mimicking a rasmussen aneurysm. Ochsner J 2018;18:104-7.

2. Skiada A, Lass-Floerl C, Klimko N, Ibrahim A, Roilides E, Petrikkos G. Challenges in the diagnosis and treatment of mucormycosis. Med Mycol 2018;56(Suppl_1):93-101. 